\title{
The Efficacy of the Echelon 10 Pre-Shaped Microcatheter for Delivering a Stent in Difficult Conditions
}

\author{
Ryuzaburo Kanazawa ${ }^{1 *}$, Takanori Uchida ${ }^{1}$, Tetsuhiro Higashida ${ }^{1}$, Yuichi Takahashi ${ }^{1}$, Naoyuki \\ Arai $^{2}$, Hidenori Ohbuchi' and Tomoyuki Yoshihara ${ }^{3}$
}

${ }^{1}$ Department of Neurosurgery, Nagareyama Central Hospital, Japan

${ }^{2}$ Department of Neurosurgery, Tokyo Women's University East, Japan

${ }^{3}$ Department of Emergency and Critical Care Medicine, Kansai Medical University General Medical Center, Japan

*Corresponding author: Ryuzaburo Kanazawa, M.D., Ph.D, Department of Neurosurgery, Nagareyama Central Hospital, 2-132-2 Higashi-Hatsuishi, Nagareyama-shi, Chiba, Japan, Tel: 81-4-7154-5741, Fax: 81-4-7155-0450

\begin{abstract}
Objective: In some endovascular procedures, the insertion of stent delivery catheters (SDCs) is difficult. We demonstrated the efficacy of the Echelon 10 MicroCatheter (90-degree angle; Medtronic, Dublin, Ireland) during such procedures.

Methods: We used the Echelon 10 MicroCatheter during difficult procedures to navigate SDCs to the distal artery from the aneurysm. The purpose of this was to reduce mechanical stress on the aneurysmal wall. The SDC was inserted by means of an exchange technique with an intermediate catheter to guarantee safety during the exchange.

Results: A total of 69 procedures involving stent-assisted technique for cerebral aneurysm (including 14 cases of ruptured aneurysm) were performed in our institution from November 2016 to July 2019. The Echelon 10 MicroCatheter was used in 17 cases (including 4 cases of ruptured aneurysm). Intra-aneurysmal insertion of an SDC was not needed in 10 of those 17 procedures. In residual aneurysms, it was possible to perform the operation without stress on the aneurysmal wall. In three cases of ruptured aneurysm, the embolization procedures were completed without intra-aneurysmal introduction of an SDC.
\end{abstract}

Conclusions: The Echelon 10 MicroCatheter (90-degree angle) may be useful for guiding the insertion of SDCs in select situations.

\section{Keywords}

Echelon 10 Microcatheter, Stent delivery catheter, Stent-assisted technique, Cerebral aneurysm

\section{Introduction}

Cerebral aneurysm embolization performed with a stent-assisted technique is increasingly used, because high-quality neck bridge stents are available $[1,2]$. In many aneurysms that have a broad neck or involve the parent artery and that were traditionally difficult to treat, endovascular coil embolization, or flow diverter stent placement can be performed; however, in some cases, guiding the stent delivery catheter (SDC) to the distal aspect of the aneurysm remains difficult. In this study, we investigated the efficacy of the Echelon 10 MicroCatheter (90-degree angle; Medtronic, Dublin, Ireland) in helping with the insertion of the SDC in appropriate situations.

\section{Materials and Methods}

There were 159 consecutive cerebral aneurysm embolizations performed in our institution from November 2016 to July 2019; in 69 cases (55 unruptured and 14 ruptured aneurysms), a stent-assisted technique was used. The Echelon 10 MicroCatheter (90-degree angle) was used in 17 (13 unruptured and 4 ruptured aneurysms) of the 69 procedures. The details were summarized in Table 1.

The Echelon 10 MicroCatheter was used when stent assistance was necessary and when insertion of the SDC into the artery distal from the aneurysm was difficult or when introducing the catheter around the aneurysmal

Citation: Kanazawa R, Uchida T, Higashida T, Takahashi Y, Arai N, et al. (2020) The Efficacy of the Echelon 10 Pre-Shaped Microcatheter for Delivering a Stent in Difficult Conditions. Neurosurg Cases Rev 3:054. doi.org/10.23937/2643-4474/1710054

Accepted: December 29, 2020; Published: December 31, 2020

Copyright: (C) 2020 Kanazawa R, et al. This is an open-access article distributed under the terms of the Creative Commons Attribution License, which permits unrestricted use, distribution, and reproduction in any medium, provided the original author and source are credited. 
Table 1: Summary of cases.

\begin{tabular}{|c|c|c|c|c|c|c|c|}
\hline Case & Age & Sex & IAN & Efficacy & Location & & Stent \\
\hline 1 & 82 & $\mathrm{~F}$ & No & Yes & PCA & Unruptured & Lvis Jr \\
\hline 2 & 83 & $M$ & No & No & IC top & Unruptured & Neuroform EZ \\
\hline 3 & 83 & M & Yes & Yes & M1M2 & Unruptured & Atlas \\
\hline 4 & 65 & $\mathrm{~F}$ & No & Yes & M1M2 & Unruptured & Atlas \\
\hline 5 & 69 & M & Yes & No & M1M2 & Unruptured & Atlas \\
\hline 6 & 68 & $F$ & Yes & Yes & BA top & Unruptured & Atlas \\
\hline 7 & 67 & $\mathrm{~F}$ & Yes & Yes & M1M2 & Unruptured & Atlas \\
\hline 8 & 65 & M & Yes & Yes & BA top & Ruptured & Atlas \\
\hline 9 & 61 & $\mathrm{~F}$ & Yes & Yes & ICD & Ruptured & Atlas \\
\hline 10 & 61 & M & No & Yes & M1M2 & Unruptured & Atlas \\
\hline 11 & 84 & $F$ & No & Yes & M1M2 & Unruptured & Atlas \\
\hline 12 & 70 & $\mathrm{~F}$ & No & No & Acom & Unruptured & Atlas \\
\hline 13 & 72 & $\mathrm{~F}$ & No & No & M1M2 & Unruptured & Atlas \\
\hline 14 & 59 & $M$ & No & Yes & M1M2 & Ruptured & Atlas \\
\hline 15 & 59 & $\mathrm{~F}$ & No & Yes & M1M2 & Ruptured & Atlas \\
\hline 16 & 76 & $M$ & Yes & Yes & Acom & Unruptured & Atlas \\
\hline 17 & 66 & $F$ & No & No & ACAD & Unruptured & Atlas \\
\hline
\end{tabular}

Intra-aneurysmal navigation (IAN) along the aneurysmal wall of Echelon-10 (90) is observed in 7 cases. In the remaining 10 cases, IAN was not needed. In 12 cases, Echelon-10 was considered to be effective for stent delivery catheter introduction. ICD: IC dorsal; ACAD: ACA distal.

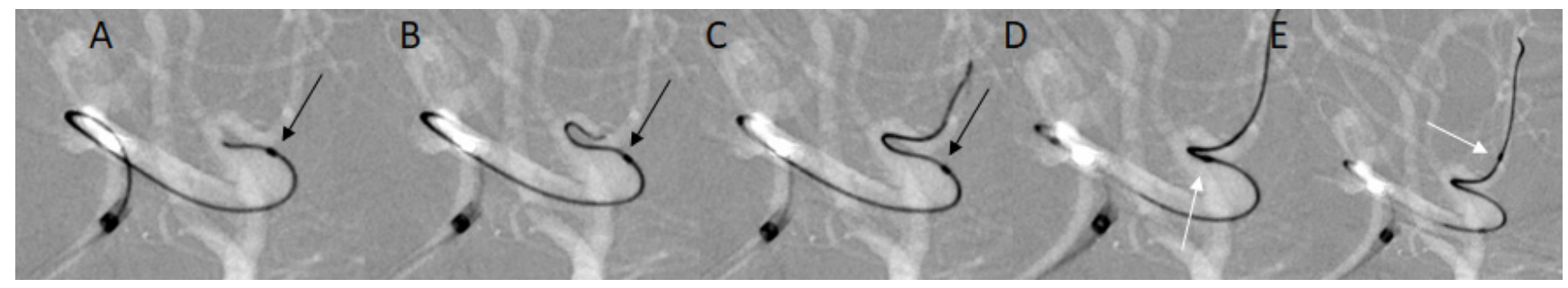

Figure 1: A case of unruptured aneurysm in the right middle cerebral artery. The microwire navigation from the Echelon 10 MicroCatheter in the aneurysm is shown. Minimum kick back of the tip (Black arrows; A, B, and C) is shown, and tracking was smooth, with lower pressure on the aneurysmal wall (White arrows; D and E).

wall seemed difficult. In most of the cases, a Neuroform Atlas stent (Stryker, Fremont, CA, USA) was chosen, and in those cases, an Excelsior XT-17 Microcatheter (Stryker) was selected as an SDC. (The Excelsior SL-10 Microcatheter [Stryker] is not used as an SDC in Nagareyama Central Hospital to avoid failure of the stent system inside the aneurysm or in the proximal artery; in two cases, the system failed in the early stage of Atlas placement). We believe that the XT-17 microcatheter may be more efficacious and safer in successful Atlas placement than the SL-10 microcatheter. After the Echelon 10 MicroCatheter using a microwire (Asahi CHIKAl, Asahi Intecc Co., Ltd., Aichi, Japan) is inserted into the distal artery, the XT-17 microcatheter, used for stent delivery, is inserted through a wire exchange technique with the use of an Asahi CHIKAI $315 \mathrm{~cm}$ EXC guidewire (Asahi Intecc Co., Ltd., Aichi, Japan). For a safer exchange procedure, we usually use an intermediate-sized catheter (Asahi Fubuki 043 [4.2-Fr]; Asahi Intecc) and navigate this catheter as close to the aneurysm as possible. In our opinion, this is important for uneventful exchange procedures.

The tip of CHIKAI $315 \mathrm{~cm}$ EXC guidewire is soft and flexible; therefore, when the distal artery is tortuous or diverges in an acute angle, this microwire is proceeded using the Echelon-10 microcatheter's support, followed by exchanging the catheter with XT-17. Following this, a trans-cell technique or jail technique was performed depending on the situation.

Use of each radiological image was approved by our ethics committee, and written informed consent was obtained from each patient.

\section{Results}

In ten cases, an intra-aneurysmal insertion technique was not necessary; in the seven cases in which this technique was used, only a minor catheter kickback phenomenon was observed during microwire (CHIKAI $315 \mathrm{~cm} \mathrm{EXC)} \mathrm{navigation,} \mathrm{and} \mathrm{the} \mathrm{stress} \mathrm{on} \mathrm{the} \mathrm{aneurys-}$ mal wall may be reduced (Figure 1). The stiffness of the 
tip of the Echelon 10 MicroCatheter seemed to facilitate insertion. In two large aneurysms (>10 mm), the flexure was released safely after intra-aneurysmal insertion of the microcatheter (Figure 2). Furthermore, in three cases of ruptured aneurysm, SDC guidance was performed without any stress on the aneurysmal wall (Figure 3). In two cases, our attempt to insert the Excelsior XT-17 Microcatheter failed. We then inserted the Echelon 10 MicroCatheter successfully (Figure 4). No thromboembolic events, no vessel injury, and no perforation of the

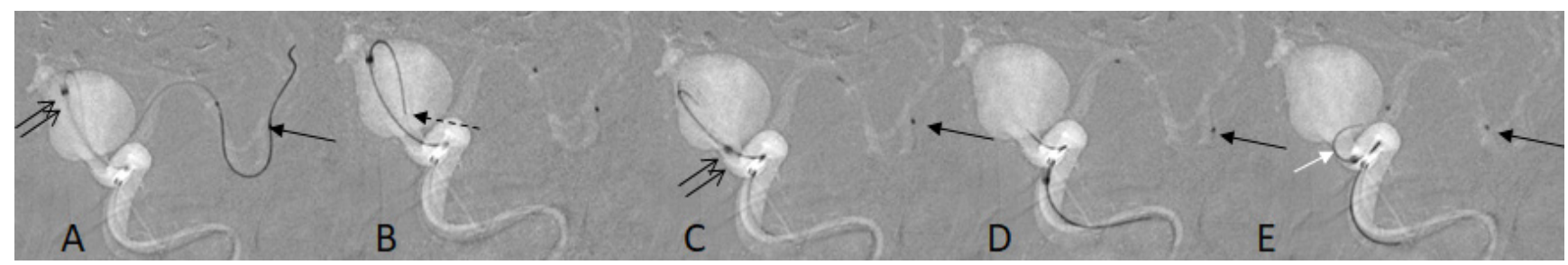

Figure 2: A case of the method of releasing the flexure of the catheter in a large aneurysm. The intermediate catheter (double arrows) was inserted near the top of the flexure (A); then the wire (Asahi CHIKAI) was pulled back to the insertion point (dotted arrow; B), and the intermediate catheter and Echelon 10 MicroCatheter (double arrows) were pulled slowly together (C and $D$ ). After releasing (white arrow), minimum transposition of the catheter tip occurred (E). The single black arrow in each image points to the tip of the Echelon 10 MicroCatheter.

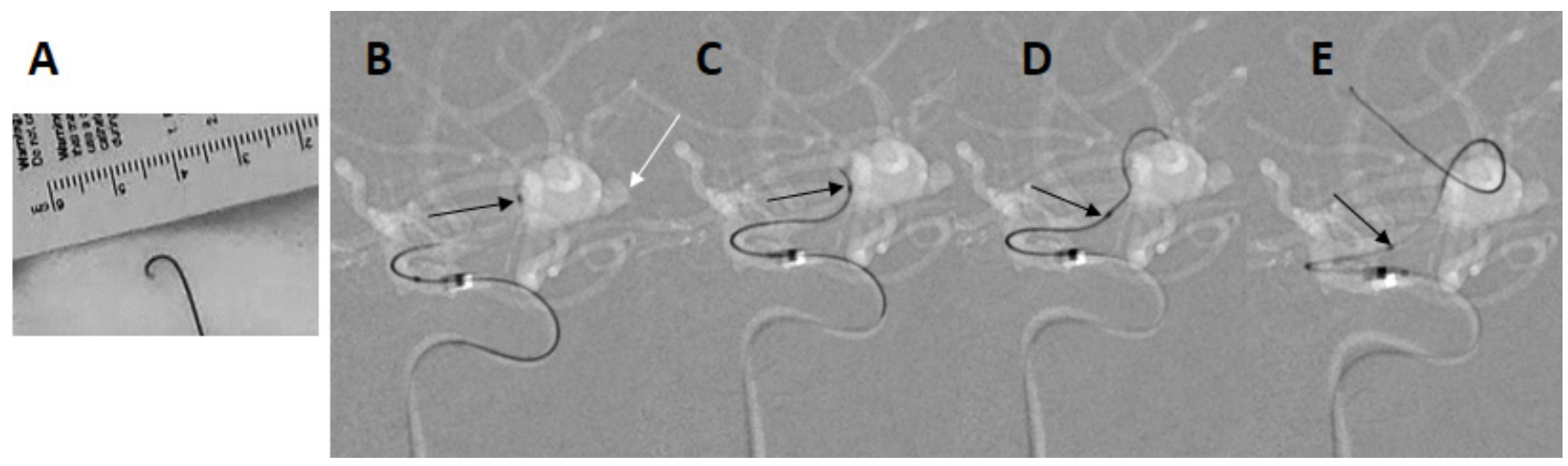

Figure 3: A case of ruptured aneurysm in the right middle cerebral artery. A bleb (White arrow in $\mathrm{B}$ ) is visible. The Echelon 10 MicroCatheter was shaped as a "J" (A), and the tip (black arrow) was inserted into the M2 trunk (B). The wire (Asahi CHIKAI $315 \mathrm{~cm} \mathrm{EXC)}$ progressed (C and D) with insignificant catheter kick back (E).
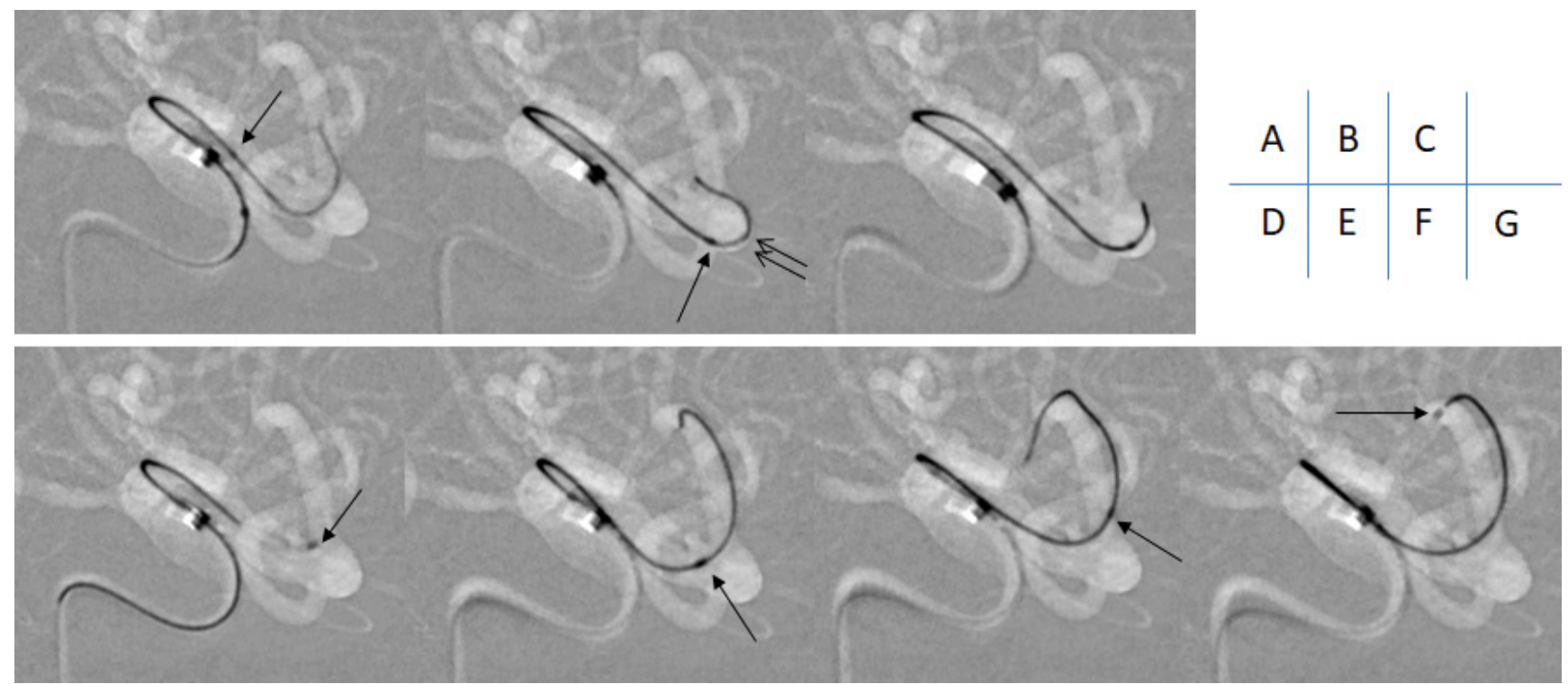

Figure 4: A case of unruptured aneurysm in the right middle cerebral artery (MCA). Comparison of the performances of the Excelsior XT-17 Microcatheter and the Echelon 10 MicroCatheter. The microwire (Asahi CHIKAI black 14 soft tip) was inserted into the upper trunk of the MCA (A), followed by tracing with the Excelsior XT-17 Microcatheter, but the microwire failed to support the catheter $(\mathrm{B}$, double arrows, and $\mathrm{C})$. The tip of the Echelon 10 MicroCatheter (D, arrow) turned toward the M2 upper trunk, supporting the wire that proceeded distally ( $E$ and $F)$, and proceeded over the wire $(G)$ without any stress on the aneurysmal wall. 
aneurysm occurred during the exchange procedures with the Asahi CHIKAI $315 \mathrm{~cm}$ EXC.

\section{Discussion}

Endovascular procedures for cerebral aneurysms are increasingly performed, because high-quality neck bridge stents are available and various other devices have been developed [1,2]. Most aneurysms formerly considered difficult to cover by endovascular technique are now treatable with stents. Endovascular coiling is as efficacious as aneurysm clipping in cases of ruptured $[3,4]$ and unruptured aneurysms, [5] and favorable results for middle cerebral aneurysms have been obtained with endovascular technique [1,6]. More aneurysms are being treated with endovascular procedures, and more tough cases are being treated, and so various devices should be used to perform safe and reliable maneuvers.

In a number of cases, advancing the SDC toward the distal vessel is difficult. Panagiotopoulos, et al. demonstrated an "intra-aneurysmal navigation technique" as a method of advancing an SDC along the aneurysmal wall [7]. Chapot, et al. reported a valuable technique for microcatheter induction without exchange maneuver ("sheeping technique"), [8] and Toyoshima, et al. reported use of the same technique [9]. Ito, et al. presented a "temporary caging technique" in which microcatheter navigation is performed after preliminary insertion of an intra-aneurysmal coil frame to support the catheter [10]. Kanenaka, et al. described a navigation technique in which a flow-directed microcatheter was used to reduce stress on the aneurysmal wall [11]. Kwon, et al. reported the suitable shaping of the tip of the microcatheter when navigation to the distal artery is difficult [12]. Wolfe, et al. demonstrated a unique method of catheter insertion with an inflated balloon into the aneurysm [13]. Each procedure is effective, depending on the situation.

The Echelon 10 MicroCatheter has a comparatively strong tip and other features that enable use of the torque-like microwire, and so it is possible to turn this catheter toward the concerned vessel and insert the microwire smoothly without significant kickback. When necessary, the manual strong J-shaped tip enables the operator to navigate into the distal segment of the artery, which diverges in an acute angle from the aneurysm and the parent artery [8]. The difference of performance between Echelon 10 MicroCatheter and other catheters shown in Figure 4 may be explained by these distinctive features. The Echelon-10 microcatheter may be used to support the microwire while navigating it to the artery distal from the aneurysm, when appropriate.

Intra-aneurysmal navigation is frequently mandatory in the treatment of large and giant aneurysms. In particular, releasing the flexure of the catheter system in giant aneurysms is not easy. Oran, et al. demonstrated the "rapid pull back technique" [14] and Sakamoto, et al. reported the "stent anchor technique" [15] as methods to use when releasing the flexure; these are effective options, but in both techniques, the whole catheter system may exit the distal artery, and recovering the catheter in such a situation is laborious. In our study, it was not easy to release the flexure in the two aneurysms that measured more than $15 \mathrm{~mm}$ in diameter, but pulling the intermediate catheter and the Echelon 10 MicroCatheter together resulted in successful release (Figure 2). The Echelon 10 MicroCatheter seemed to be effective for such situations, and this technique was thus considered a useful option. Guidance of the microwire to the target vessel with lower pressure against the aneurysmal wall was possible during intra-aneurysmal navigation (Figure 1).

The present study has some limitations. First, objectively estimating the concerned microcatheter's efficacy is difficult. Second, our investigation only included 17 cases. Third, the exchange maneuver is troublesome and has a risk of wire jumping, which may result in vessel perforation. We therefore introduced the support catheter close to the aneurysm to shorten the distance of the microwire out of the support catheter; following this, we have not experienced any case of wire jumping or returning into the catheter. However, as this technique may require a slightly excessive effort, we believe that it should be used in restricted situation as an alternative.

\section{Conclusion}

The wire exchange technique is necessary for SDC navigation in our method, which is a shortcoming. But we think that the risks during exchange (perforation or injury to the arterial wall or aneurysm by wire jumping) [8] can be reduced by the insertion of an intermediate catheter to the aneurysm as close as possible. Our technique may be an option for SDC navigation in stent-assisted endovascular procedures.

\section{Acknowledgment}

The authors would like to acknowledge the radiologists at their hospital for their dedicated assistance during the procedures and Enago for the English language review.

\section{Disclosure of Interest}

The authors declare no conflicts of interest associated with this manuscript.

\section{References}

1. Zhang L, Wang H, Pan $Y$, Mao L (2019) Effect analysis of microsurgical clipping and endovascular embolization for the treatment of middle cerebral artery aneurysms. World Neurosurg

2. Russo R, Bradac GB, Castellan L, Ivan G, Diego G, et al. (2020) Neuroform Atlas stent-assisted coiling of ruptured intracranial aneurysms: A multicenter study. J Neuroradiol. 
3. Molyneux A, Birks J, Clarke A, Mary S, Richard S (2015) The durability of endovascular coiling versus neurosurgical clipping of ruptured cerebral aneurysms: 18 year follow-up of the UK cohort of the international subarachnoid aneurysm trial (ISAT). Lancet.

4. Molyneux AJ, Kerr RS, Yu LM, Mike C, Mary S, et al. (2005) International subarachnoid aneurysm trial (ISAT) of neurosurgical clipping versus endovascular coiling in 2143 patients with ruptured intracranial aneurysms: A randomized comparison of effects on survival, dependency, seizures, rebleeding, subgroups, and aneurysm occlusion. Lancet.

5. Jiang Z, Chen Y, Zeng C, Jiugeng F, Yilv W, et al. (2020) Neurosurgical clipping versus endovascular coiling for patients with intracranial aneurysms: A systematic review and meta-analysis. World Neurosurg 138: e191-e222.

6. Schwartz C, Aster HC, Al-SchameriR, E Muller-Thies-Broussalis, C Griessenauer, et al. (2018) Microsurgical clipping and endovascular treatment of middle cerebral artery aneurysms in an interdisciplinary treatment concept: Comparison on long-term results. Interv Neuroradiol 24: 608-614.

7. Panagiotopoulas V, Gizewski E, Wanke I (2008) Intra-aneurysmal navigation technique for stenting of a wide-neck basilar aneurysm with a new self-expandable stent: Technical note. Minim Invasive Neurosurg 51: 240-243.

8. Chapot R, Nordmeyer H, Heddier M, Velasco A, P Schooss, et al. (2013) The sheeping technique or how to avoid exchange maneuvers. Neuroradiology 55: 989-992.

9. Toyoshima A, Sugiu K, Tokunaga H, T Shimizu, J Haruma, et al. (2013) Usefulness of the "sheep technique" in the en- dovascular treatment for cerebral aneurysms. J Neurovasc Ther 7: 3-10.

10. Ito H, Onodera H, Wakui D, Uchida M, T Sase, et al. (2015) The "temporary caging" technique for catheter navigation in patients with intracranial wide-necked aneurysms. Int J Clin Exp Med 8: 11214-11219.

11. Kanenaka N, Sato H, Abe H, Koike T, K Torihashi, et al. (2014) Exchange technique using flow directed microcatheter during difficulty in navigation of delivery catheter in stent-assisted coil embolization. J Neurovasc Ther 8: 107113.

12. Kwon BJ, Seo DH, Ha YS, Lee KC (2012) Endovascular treatment of wide-necked cerebral aneurysms with an acute angle branch incorporated into the sac: Novel methods of branch access in 8 aneurysms. Neurointervention 7: 93-101.

13. Wolfe SQ, Farhat H, Moftakhar R, M Samy Elhammady, M Ali Aziz-Sultan (2011) Intraaneurysmal balloon assistance for navigation across a wide-necked aneurysm. J Neurosurg 112: 1222-1226.

14. Oran L, Cinar C, Bozkaya H, Besir FH (2013) The rapid pull-back technique for navigation across a wide-necked aneurysm. Interv Neuroradiol 19: 16-20.

15. Sakamoto S, Shibukawa M, Tani I, Shuichi Oki, Kaoru Kurisu (2016) Open-cell stent deployment across the wide neck of a large middle cerebral aneurysm using the stent anchor technique. J Cerebrovasc Endovasc Neurosurg 18: 38-41. 